\title{
MARQUAGE MAGNÉTIQUE INTERNE : ESSAIS DE TOLÉRANCE PAR DE JEUNES SAUMONS ATLANTIQUES (SALMO SALAR L.)
}

\author{
J. DUMAS (1), P. PROUzeT (2)
}

(1) Institut National de la Recherche Agronomique - Centre de Recherches Hydrobiologiques - Laboratoire d'Écologie des Poissons et d'Aménagement des Pêches - BP 3 - SAINT PEE SUR NIVELLE - 64310 ASCAIN (France)

(2) Centre Océanologique de Bretagne - C.N.E.X.O. BP 337 - 29273 BREST CEDEX (France)

\section{RÉSUMÉ}

Les effets de l'implantation d'une marque magnétique "Metalimphy" * de $20 \times 1,5 \mathrm{~mm}$ dans la cavité abdominale de jeunes saumons d'élevage sont comparés à ceux d'un marquage classique par ablation de la nageoire adipeuse. Les essais sont effectués sur des tacons de deuxième été et des pré-saumoneaux d'un an dans deux piscicultures de Bretagne et des Pyrénées-Atlantiques.

Quel que soit le mode d'insertion pratiqué (ventral ou latéral) cette technique n'a aucun effet significatif sur la survie et la croissance des poissons, qu'ils soient tacons (observés sur 194 jours) ou pré-saumoneaux (observés sur 56 jours). La cicatrisation est rapide et ce marquage n'entrave pas la smoltification. L'emploi d'une poudre antibiotique cicatrisante ne semble pas nécessaire en conditions normales de pisciculture.

L'insertion par voie ventrale s'avère préférable à l'insertion latérale; elle n'entraîne pas de pertes de marques et se révèle plus facile à pratiquer. migration.

Mots clés: Saumon atlantique, marquage magnétique, survie, croissance,

\section{SUMMARY}

Internal magnetic tagging: tests of tolerance by Atlantic salmon juveniles (Salmo salar L.).

The effects ptoduced by the introduction of a magnetic tag "Metalimphy" $\left({ }^{*}\right)$ $(20 \times 1.5 \mathrm{~mm})$ into the abdominal cavity of hatchery-reared young salmon are compared to those obtained by a classical marking by removal of the adipose fin. The experiments are carried out on second summer parrs or on 1 year-old pre-smolts in two fish farms in Brittany and Pyrénées Atlantiques.

Whatever the method of insertion utilized (ventral or lateral, this technique has no significant effect on survival and growth of fish, whether they be parr (observed during 194 days) or pre-smolt (observed during 56 days). Healing is quick and this tagging method does not delay smoltification. The utilization of a healing antibiotic powder does not seem necessary, under normal conditions in hatcheries.

The ventral insertion is more advisable than the lateral one; it does not involve tag losses and is easier to make.

Key-words : Atlantic salmon, magnetic tagging, survival, growth, migration.

\footnotetext{
* Metalimphy: IMPHY S.A., 168 rue de Rivoli, 75001 PARIS, France.
} 


\section{INTRODUCTION}

Nombreux sont les types de marquages pratiqués sur les juvéniles de Saumon atlantique. Chacun d'eux présente des avantages et des inconvénients variés.

Les marquages individuels par étiquettes dorsales (CARLIN, 1955) réduisent considérablement le taux de survie des poissons (SAUNDERS et ALLEN, 1967). Les ablations de nageoires ou le cryomarquage, dont l'influence est réduite ou nulle sur la survie, ne permettent d'identifier qu'un nombre restreint de lots de poissons (SAUNDERS et ALLEN, 1967 ; PIGGINS, 1972; REFSTIE et AULSTAD, 1975 ; DUMAS, 1977 et 1978); de plus, ces techniques perdent de leur efficacité par manque de lisibilité de la marque après un ou deux ans. Enfin, les marques magnétiques nasales (ISAKSSON et BERGMAN, 1977), bien que peu traumatisantes et d'une bonne tenue, font obligation de sacrifier les sujets pour extraire et décoder les marques.

Une solution intéressante est proposée par HARACHE, LAGARDE et PROUZET (1978) avec une marque magnétique interne, introduite dans la cavité générale de Saumons coho (Oncorhynchus kisutch). Ces inserts peuvent être détectés et décodés à distance en faisant passer les poissons vivants dans une boucle de solenoïde; cette dernière peut être immergée dans une passe à poissons. Actuellement, ce procédé permet d'identifier une quinzaine de lots différents (ANONYME, 1979).

Si au plan technique la confection d'inserts de ce type et de leur appareil d'identification est réalisable, il est néanmoins nécessaire de vérifier la tolérance de ces marques par de jeunes saumons atlantiques, réputés pour leur fragilité.

L'incidence des marques sur la survie et la croissance est testée lors de deux essais sur des juvéniles de pisciculture à différents stades.

Ce travail fait l'objet d'une collaboration entre le Centre National pour I'Exploitation des Océans (C.N.E.X.O.) et l'Institut National de la Recherche Agronomique (I.N.R.A.).

\section{MATÉRIEL ET MÉTHODE}

\subsection{Principe}

Deux séries d'observations sont conduites dans deux piscicultures différentes.

Dans un premier essai, les effets de deux voies d'insertion des marques dans la cavité générale de pré-saumoneaux $d$ 'un an (lots $A$ et $B$ ) sont comparés à un marquage classique par ablation de la nageoire adipeuse (lot témoin C) depuis l'époque habituelle du marquage jusqu'au stade saumoneau prêt pour l'avalaison. Cette étude a lieu du 7 février au 3 avril 1980 au domaine expérimental de I'I.N.R.A. à St-Pée-sur-Nivelle (Pyrénées Atlantiques).

Lors d'un deuxième essai, l'incidence de l'utilisation ou non d'un antibiotique (lots $\mathrm{D}$ et $E$ ) chez des tacons dans leur deuxième été subissant une même voie d'insertion, est comparée comme précédemment à un marquage par ablation de la nageoire adipeuse (lot témoin $\mathrm{F}$ ). Ces observations effectuées à la pisciculture expérimentale du C.N.E.X.O. au Quìnquis, sur le bassin de I'Elorn (Finistère), s'étendent sur une période plus longue, du 13 août 1980 au 23 février 1981 .

\subsection{Les marques}

Les inserts utilisés sont des marques inertes (sans brin d'alliage magnétique), analogues aux inserts magnétiques déjà décrits par HARACHE, LAGARDE et PROUZET (1978) et ANONYME (1979). Ce sont des cylindres de $20 \times 1,5 \mathrm{~mm}$ aux extrémités arrondies, en résine moulée, bien tolérée par les tissus organiques; leur poids est très faible: $0,04 \mathrm{~g}$. Ils sont de couleur différente pour chacun des deux lots traités et en observation dans une même pisciculture (vert pour l'un et bleu pour l'autre).

\subsection{Mode d'insertion des marques}

Lors du premier essai, les inserts sont introduits dans la cavité générale des poissons du lot $A$ par voie ventrale et par voie latérale pour ceux du lot B (fig. 1). Dans la deuxième expérience, les sujets des lots $D$ et $E$, subissent une insertion latérale, comme ceux du lot B (fig. 1).

Après anesthésie au phenoxy-2-éthanol $(2 \mathrm{ml} / 5 \mathrm{l}$ d'eau), une incision est pratiquée à l'aide d'une aiguille hypodermique creuse à biseau long de $1,5 \mathrm{~mm}$ de 


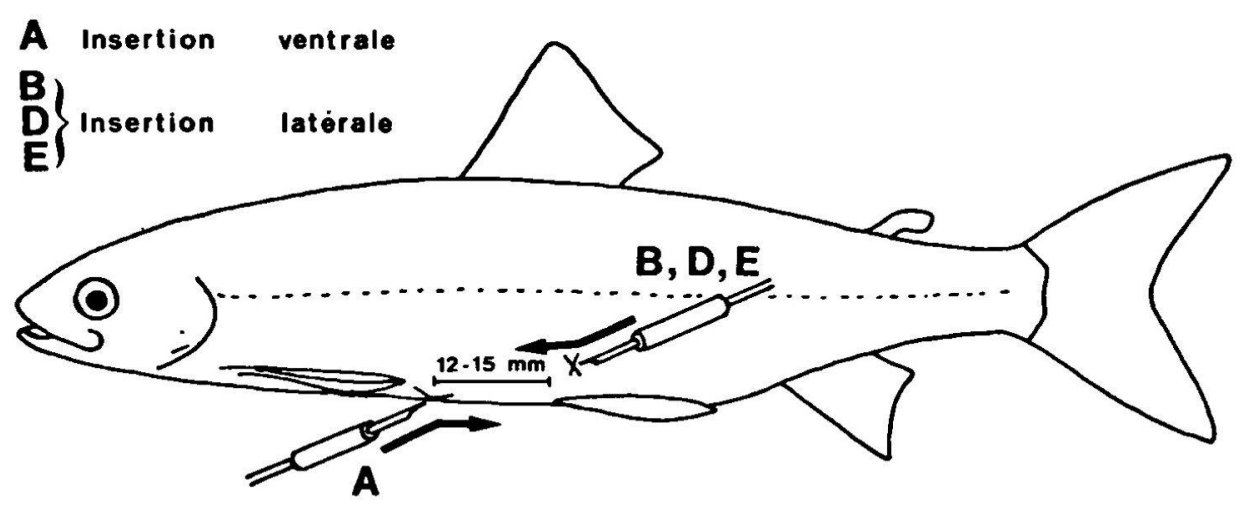

Figure 1: Points d'insertion de marques magnétiques dans la cavité abdominale de jeunes saumons pour les lots A, B, D et E.

diamètre extérieur et $40 \mathrm{~mm}$ de longueur, montée sur une seringue de $1 \mathrm{ml}$ servant de manche (CHEVASSUS, comm. pers.) *. Un manchon de tube en matière plastique souple est placé sur l'aiguille à $9 \mathrm{~mm}$ de la pointe pour éviter d'enfoncer exagérément celle-ci dans la paroi abdominale du poisson. Un jeu de 6 aiguilles ainsi montées trempent dans de l'alcool à $95^{\circ}$ et sont utilisées à tour de rôle.

Dans le cas de l'insertion ventrale (lot $A$ ), le point de pénétration se situe à une distance de 12 a $15 \mathrm{~mm}$, selon la taille du poisson, en avant de l'attache des nageoires pelviennes; I'aiguille est enfoncée à $45^{\circ}$ en direction de l'arrière, sur 2 à $3 \mathrm{~mm}$ de profondeur, puis parallèlement à l'axe du corps pour ne pas léser l'intestin (fig. 1 et 2). Dans le cas de l'insertion latérale (lots B, D et E), l'aiguille est enfoncée un peu au-dessus de la base des nageoires pelviennes à $45^{\circ}$ en direction oblique avant sur 4 a $5 \mathrm{~mm}$, puis redressée parallèlement à la paroi abdominale dans l'axe du corps (fig. 1).

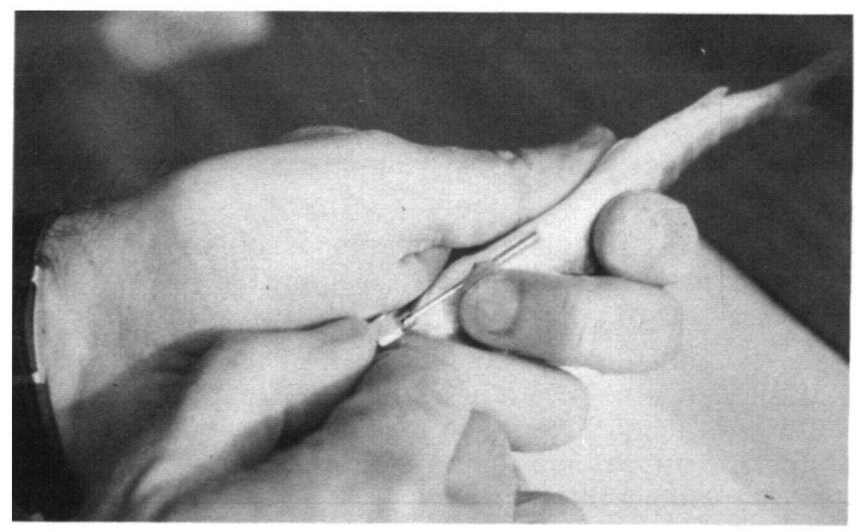

Figure 2: Perforation ventrale de la paroi abdominale d'un jeune saumon de $14 \mathrm{~cm}$ à l'aide d'une aiguille hypodermique de $1,5 \mathrm{~mm}$ de diamòtre. Après avoir pénétré de 2 a $3 \mathrm{~mm}$ avec un angle de $45^{\circ}$ vers l'arrière permettant à la pointe d'atteindre la cavité générale, l'axe de l'aiguille est ensuite positionné parallèlement à la paroi abdominale; l'aiguille est enfoncée de 3 a $4 \mathrm{~mm}$ de plus pour achever I'incision.

Les inserts stérilisés au préalable par immersion de quelques minutes dans de l'alcool sont placés dans de la poudre antibiotique cicatrisante et adhésive CICATRIN **, sauf pour le lot $\mathrm{E}$ du deuxième essai. Chaque marque est saisie au moyen d'une pince à

\footnotetext{
* CHEVASSUS B., Laboratoire de Physiologie des Poissons, C.N.R.Z. Domaine de Vilvert,
} 78350 JOUY EN JOSAS.

* CICATRIN : produit fabriqué à Londres par Calmic Medical Division, the Welcome Foundation Ltd. 
dissection stérilisée et introduite dans l'abdomen en direction de l'arrière du corps pour le lot A (fig. 3), ou de l'avant pour les lots B, D et E.

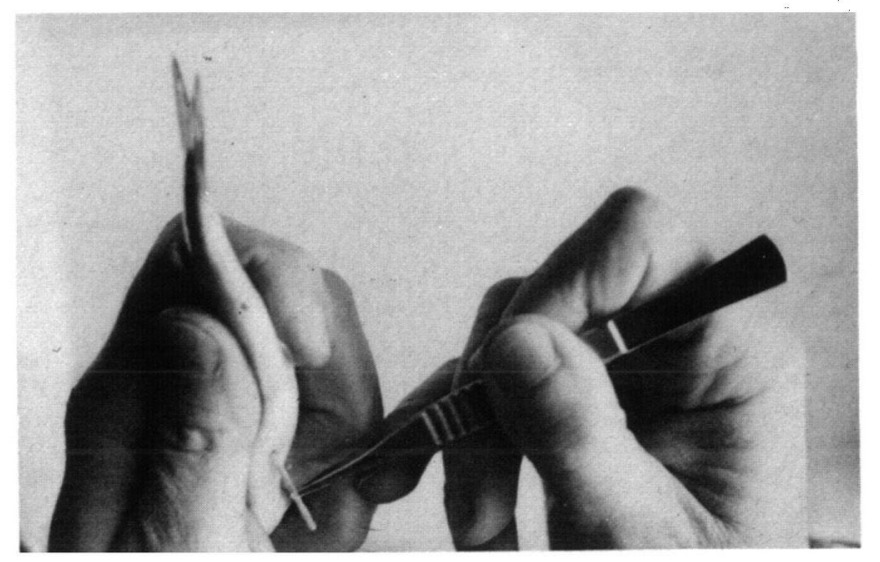

Figure 3 : Insertion d'une marque magnétique de $20 \times 1,5 \mathrm{~mm}$ après incision ventrale. La marque, ici enrobée de poudre antibiotique cicatrisante, est maintenue à l'aide d'une pince. Elle est introduite dans le même sens que l'aiguille servant à la perforation.

\subsection{Constitution des lots, mensurations, poursuite de l'élevage}

\section{Essai $n^{\circ} 1$}

Les pré-saumoneaux choisis sont tous issus d'une même groupe d'élevage (chenal de production semi-intensive) et trois lots sont individualisés le 7/02/1980.

Leur nombre est de 65 pour chacun des lots $A$ et $B$, et 130 pour le lot témoin $C$. Les poissons, à jeûn depuis $24 \mathrm{~h}$, sont pris au hasard dans une gamme de tailles de 110 à $149 \mathrm{~mm}$. Ils sont anesthésiés, mesurés au millimètre près (longueurs à la fourche), puis marqués.

Une fois ranimés, tous les sujets d'un même lot sont pesés en groupe au gramme près. Ils subissent ensuite un bain au vert de malachite ( 30 secondes au $1 / 15000$ ème).

Les trois lots sont maintenus dans un même compartiment de chenal de libération de même type que leur milieu de production $(20 \times 1,2 \times 0,3 \mathrm{~m}$, débit $15 \mathrm{l} / \mathrm{s})$ où ils sont nourris à satiété dès le lendemain de leur marquage. Ces poissons seront observés sur une période de 56 jours jusqu'à l'époque normale de smoltification et de libération $(3 / 04 / 1980)$. Un calendrier des mortalités est tenu. Les poissons morts sont mis au congélateur pour observations ultérieures.

En fin d'expérience, les jeunes saumons sont anesthésiés, dénombrés, mesurés $(\mathrm{mm})$ et pesés individuellement $(\mathrm{dcg})$. Les sujets des lots $A$ et $B$ sont sacrifiés puis disséqués pour contrôler la position de l'insert dans le corps du poisson et des lésions éventuelles.

\section{Essai n०2}

Les tacons expérimentaux sont issus d'un même bassin à courant hélicoïdal (type suédois) de $16 \mathrm{~m}^{2}$. Deux jours avant le marquage, ils sont mis à jeûn. Le lot $\mathrm{D}$ est constitué de 64 poissons, le lot $E$ de 65 , tandis que le lot témoin $F$ compte 131 sujets.

Les poissons sont ensuite manipulés comme dans le premier essai, toutefois ils ne subissent aucune pesée et le lot $E$ est opéré sans Cicatrin. Ils sont ensuite traités par un bain de Furanace.

Les trois groupes sont mélangés et élevés pendant 194 jours jusqu'au 23/02/1981 dans un bassin suédois de $4 \mathrm{~m}^{2}$. A cette date, les sujets sont contrôlés comme dans l'essai précédent, mais non pesés; les saumons des lots $D$ et E sont sacrifiés et disséqués. 


\section{RÉSULTATS}

\subsection{Essai no 1}

3.11. Survie

Après 8 semaines d'observations, le nombre de poissons survivants est 62 pour le lot $A(95,4$ p. 100), 64 dont 3 sans leur marque pour le lot $B(98,5$ p. 100) et 128 pour le lot $C$ $(98,5$ p. 100). Le pourcentage de poissons vivants avec leurs marques est respectivement de $95,4,93,8$ et 98,5 p. 100 pour chacun des groupes (tabl. 1 ) ; 4,6 p.100 des poissons du lot $B$ (insertion latérale) survivent après la perte de leurs inserts. La différence des taux de survie est faible et $n$ 'est pas significative (test $2 \hat{i}$ sur les effectifs).

Les quelques mortalités intervenues pendant la période d'observation ne semblent pas dues au mode de marquage.

\subsection{Croissance}

En début d'expérience, les longueurs moyennes à la fourche des pré-saumoneaux de chacun des lots ne diffèrent pas significativement (test de comparaison des moyennes) et leurs poids moyens sont très voisins (tabl. 2).

Leur accroissement linéaire et pondéral en 56 jours est très élevé (tabl. 2): $19,5 \mathrm{~mm}$ pour $8,4 \mathrm{~g}$ (lot A), $20,9 \mathrm{~mm}$ pour $9,4 \mathrm{~g}$ (lot B) et $20,2 \mathrm{~mm}$ pour $9,1 \mathrm{~g}$ (lot C). Les tailles moyennes de ces poissons respectivement de $146,9 \mathrm{~mm}, 148,9 \mathrm{~mm}$ et $147,0 \mathrm{~mm}$ pour chacun des lots (tabl.2), ne sont pas significativement différentes (test de comparaison des moyennes). La croissance des saumons possédant des inserts magnétiques semble se poursuivre au même rythme que celle des individus marqués par ablation de la nageoire adipeuse, donc très proche de la normale.

L'embonpoint des poissons de chaque lot mesuré par le coefficient de condition $k$ $(=100 \times$ poids en gramme/cube de la longueur en $\mathrm{cm}$ ) est d'ailleurs très voisin, $k=0,97$ à 0,98 (tabl. 2).

\subsection{Smoltification}

La totalité des sujets de chaque groupe a smoltifié au moment de l'arrêt des observations; ces jeunes saumons se trouvent au stade où ils doivent normalement être libérés sous peine de mortalités excessives. Le marquage interne ne paraît avoir aucun effet sur leurs transformations physiologiques.

\subsection{Position ou rejet des marques}

Lors du contrôle final, la cicatrisation du point d'insertion de la marque est complète sur l'ensemble des poissons des deux groupes et seule la cicatrice externe reste encore visible.

Aucun rejet de marque n'est constaté parmi les survivants du lot marqué ventralement. Par contre, trois individus marqués latéralement (4,9 p.100), possédant une cicatrice latérale bien visible, ont perdu leurs marques. Ce fait est à mettre en liaison avec la position de certains inserts découverts dans la musculature latérale de la ceinture abdominale (4,9 p.100) uniquement dans ce groupe (tabl. 1); il est dû à une moindre facilité de perforation de la paroi musculaire, plus épaisse à ce niveau que sur la face ventrale, et certaines marques ne se trouvent pas complètement engagées dans la cavité générale. Au total, près de 10 p.100 des poissons du lot B ont subi une mauvaise implantation de l'insert.

La plupart des marques se positionnent près de l'intestin antérieur quel que soit le point d'insertion: 77,4 et 57,4 p. 100 des sujets des lots $A$ et $B$ (tabl. 1). Toutefois, des inserts sont retrouvés dans la région antérieure du corps, entre les caeca pyloriques, en proportion plus importante chez les poissons incisés latéralement $(37,5 \mathrm{p} .100$ pour le lot $B$ et 22,6 p. 100 pour le lot A), ce qui peut être un risque de blessure d'organes vitaux (cœur, estomac, caeca, foie). Cependant, aucune lésion d'organe ou irritation ne sont constatées. En particulier, aucune marque n'est retrouvée dans la vessie natatoire, contrairement à ce qu'avaient constaté HARACHE, LAGARDE et PROUZET (1978) chez quelques individus incisés latéralement.

\subsection{Essai n०2}

\subsection{Survie}

A la 28 ème semaine d'observation, le nombre de survivants est de 63 , dont 6 sans leurs marques parmi les poissons du lot $D$, de 64 dont 6 sans leurs inserts pour le lot $E$, et de 128 pour le lot témoin (tabl. 3). 


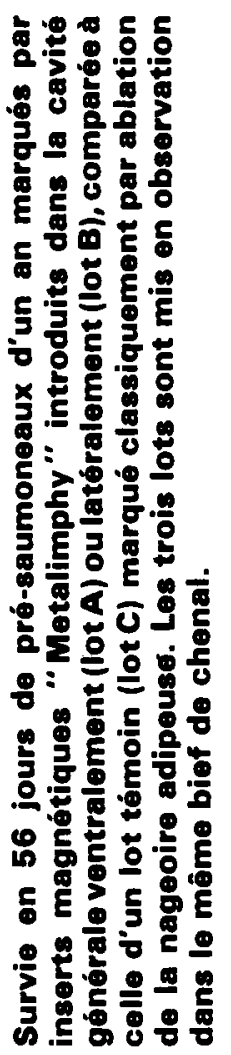

\begin{tabular}{|c|c|c|c|c|}
\hline \multicolumn{2}{|c|}{ 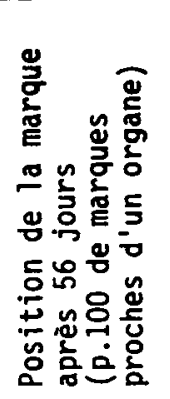 } & 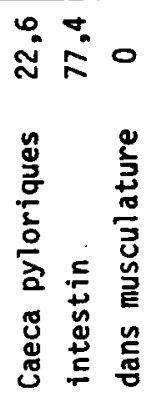 & 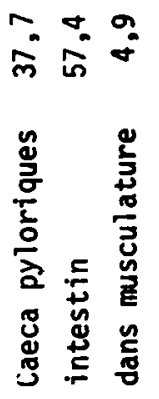 & 1 \\
\hline \multicolumn{2}{|c|}{ 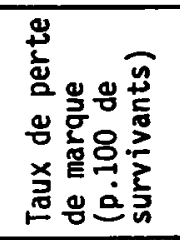 } & 0 & go & ' \\
\hline \multirow{2}{*}{ 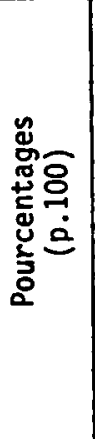 } & 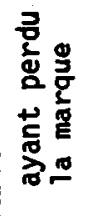 & 0 & $\stackrel{\circ}{\circ}$ & 1 \\
\hline & $\begin{array}{l}\text { y } \\
\text { g } \\
\text { o } \\
\text { प } \\
\vdots \\
\vdots\end{array}$ & मे & $\begin{array}{l}\infty \\
\infty \\
\delta\end{array}$ & $\begin{array}{l}\text { L̊ } \\
\text { o̊ }\end{array}$ \\
\hline \multirow{2}{*}{ 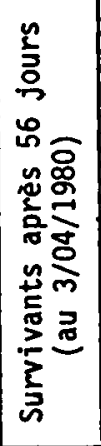 } & 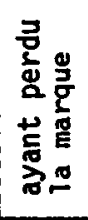 & 0 & $m$ & I \\
\hline & 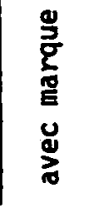 & $\tilde{\sigma}$ & $\overrightarrow{0}$ & 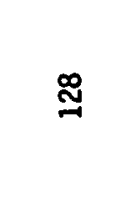 \\
\hline \multicolumn{2}{|c|}{ 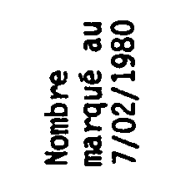 } & ณี & 绐 & 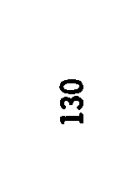 \\
\hline \multicolumn{2}{|c|}{ 范 } & 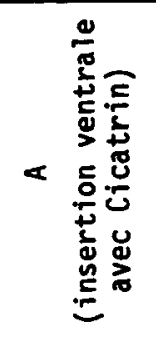 & 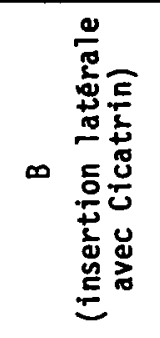 & 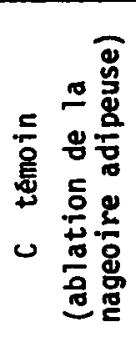 \\
\hline
\end{tabular}




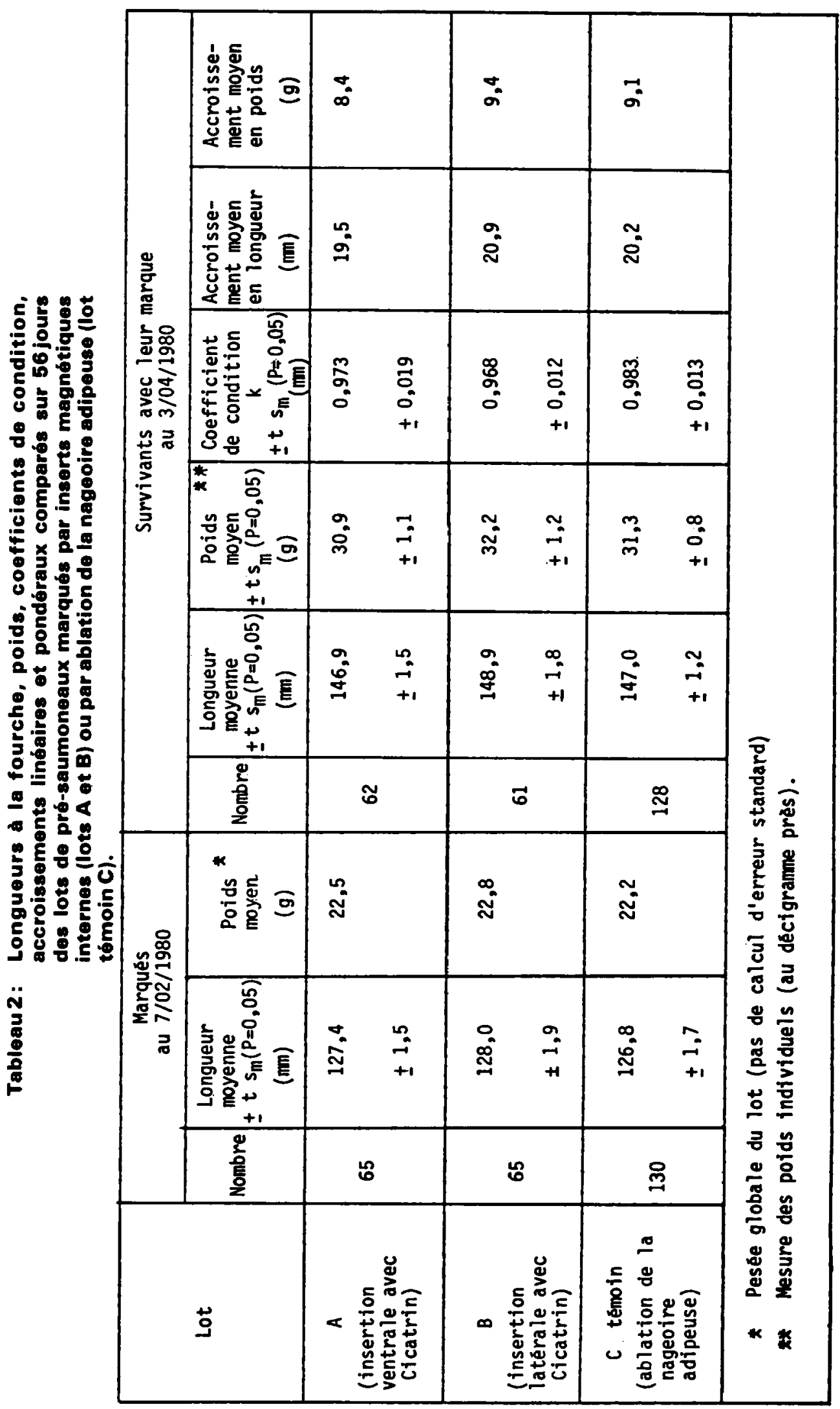




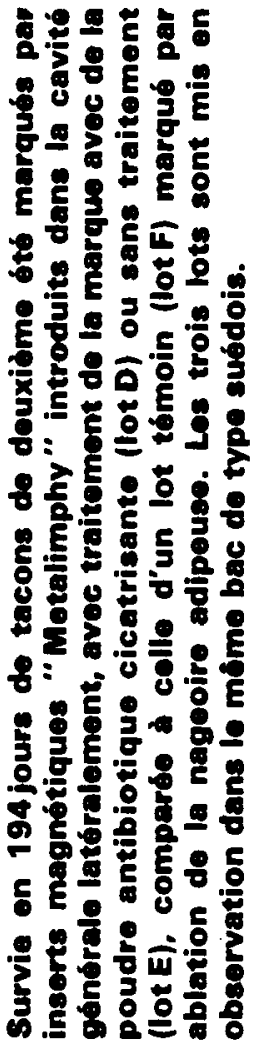

\begin{tabular}{|c|c|c|c|c|}
\hline \multicolumn{2}{|c|}{ 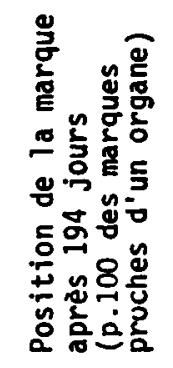 } & 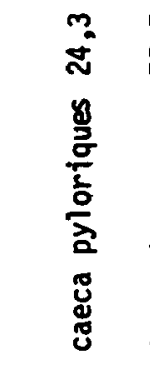 & 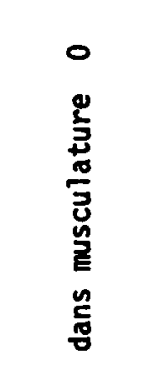 & ' \\
\hline \multicolumn{2}{|c|}{ 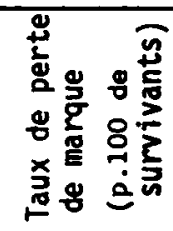 } & & & ' \\
\hline \multirow{2}{*}{ 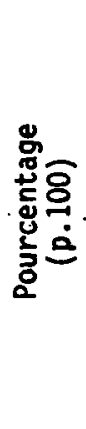 } & 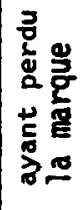 & & & ' \\
\hline & 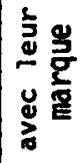 & & & $\hat{a}$ \\
\hline \multirow{2}{*}{ 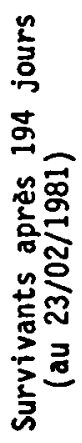 } & 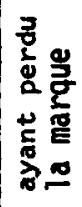 & $\boldsymbol{0}$ & 6 & ' \\
\hline & 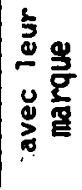 & $\sqrt{n}$ & 曲 & $\underset{\Im}{\mathbb{Z}}$ \\
\hline \multicolumn{2}{|c|}{ 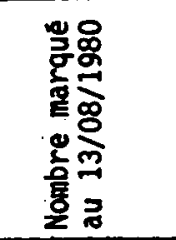 } & \multicolumn{2}{|c|}{ స్తి } & $\vec{m}$ \\
\hline \multicolumn{2}{|c|}{ 苛 } & 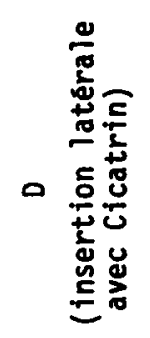 & 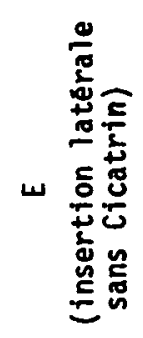 & 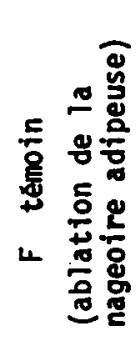 \\
\hline
\end{tabular}


Qu'elle soit effectuée avec ou sans poudre antibiotique, la pose d'inserts magnétiques n'a aucune incidence significative sur la survie des tacons (98,4 et 98,5 p.100 pour les lots $D$ et $E, 97,7$ p. 100 pour le lot témoin).

Une analyse globale des données des groupes $D$ et $E$, marqués latéralement, indique que sur une période prolongée, le pourcentage de survie des poissons avec leurs marques atteint 89,2 p.100, tandis que 9,3p.100 des sujets des mêmes lots survivent après avoir perdu leurs inserts (tabl. 3 ).

\subsection{Croissance}

Les tailles moyennes des saumons des lots marqués et du lot témoin ne diffèrent pas significativement au début des observations (tabl. 4). L'accroissement linéaire moyen en 194 jours est respectivement de $13.7 \mathrm{~mm}$ pour les lots $D$ et $E$ et de $15,9 \mathrm{~mm}$ pour le lot $F$. En fin d'expérience, les tailles obtenues, 139,2 mm pour les poissons possédant un insert et $141,1 \mathrm{~mm}$ pour ceux marqués par simple ablation, ne sont pas significativement différentes (tabl. 4).

\subsection{Position ou rejet des marques}

La cicatrisation de la lésion abdominale latérale due à l'insertion est totale, que la marque ait été traitée avec de la poudre cicatrisante (lot D) ou non (lot E).

Généralement, la marque se trouve parallelement à l'intestin dans sa portion médiane (75,7 p. 100 des sujets marqués); 24,3 p. 100 sont placées en avant de la zone d'introduction, au voisinage des caeca pyloriques et sont chez les mâles, enrobées de laitance.

La perte de marque, 9,5 p. 100 de survivants, est relativement importante (tabl. 3); elle est de 4,3p.100 chez les máles et de 15,2 p.100 chez les femelles, mais cette proportion n'est pas significativement différente $\left(\mathcal{X}^{2}\right.$ non significatif).

\section{DISCUSSION ET CONCLUSION}

Pratiqué au stade tacon ou pré-saumoneau, le marquage magnétique interne est bien toléré par les jeunes saumons d'élevage à la taille habituelle du marquage (1 10 à $149 \mathrm{~mm}$ à la fourche). Quelle que soit la voie d'insertion choisie, ventrale ou latérale, leur taux de survie n'est pas différent de celui de leurs congénères ayant subi une ablation de la nageoire adipeuse. Ces résultats confirment les observations de HARACHE, LAGARDE et PROUZET (1978) obtenues sur des saumons coho plus gros (de 30 a $60 \mathrm{~g}$ ) et effectuées sur une période plus courte ( 30 jours) en eau stérilisée; ils concordent aussi avec les résultats obtenus par CHEVASSUS (comm. pers.) sur des truites arc-en-ciel de 117 à $308 \mathrm{~g}$ marquées de la même manière.

L'incidence de ce marquage sur la croissance ou la smoltification est nulle ou négligeable; tous les lots possédant des inserts présentent des accroissements linéaires ou pondéraux très voisins de ceux des lots témoins. L'embonpoint, vérifié sur des saumoneaux prêts à migrer, est normal pour des sujets de ce stade et ne traduit aucune gêne.

L'utilisation de poudre antibiotique cicatrisante (Cicatrin) ne semble pas nécessaire dans les conditions normales de pisciculture. La cicatrisation de la plaie est complète, même dans le cas de contrôle après une période courte (56 jours) et les inserts ne paraissent engendrer aucune irritation ou lésion d'organes. Les saumoneaux peuvent ainsi être libérés sans aucune blessure en cours d'évolution.

Les pertes ou malpositions de marques constatées chez les poissons incisés latéralement (près de 10 p. 100 du lot $B$ et 9,5 p. 100 des lots $D$ et $E$ ) incitent à pratiquer l'insertion ventrale (lot A). La pénétration de l'aiguille dans une paroi musculaire mince est plus facile et plus fiable : cette voie n'occasionne pas d'introduction incomplète de marques ou d'insertions dans la musculature, vraisemblablement à l'origine des pertes de marques pendant la période de cicatrisation.

Cette technique, plus rapide que la pose d'étiquettes dorsales, permet de traiter 150 à 180 poissons par heure (un opérateur entrainé devrait doubler ce rythme). Bien que plus difficile et plus lente a mettre en œuvre que le cryomarquage (DUMAS, 1977), elle offre l'avantage d'une individualisation efficace et rapide d'un nombre plus important de lots (une quinzaine); d'autre part, le sacrifice de l'animal n'est pas nécessaire comme dans le cas du marquage magnétique nasal (ISAKSSON et 

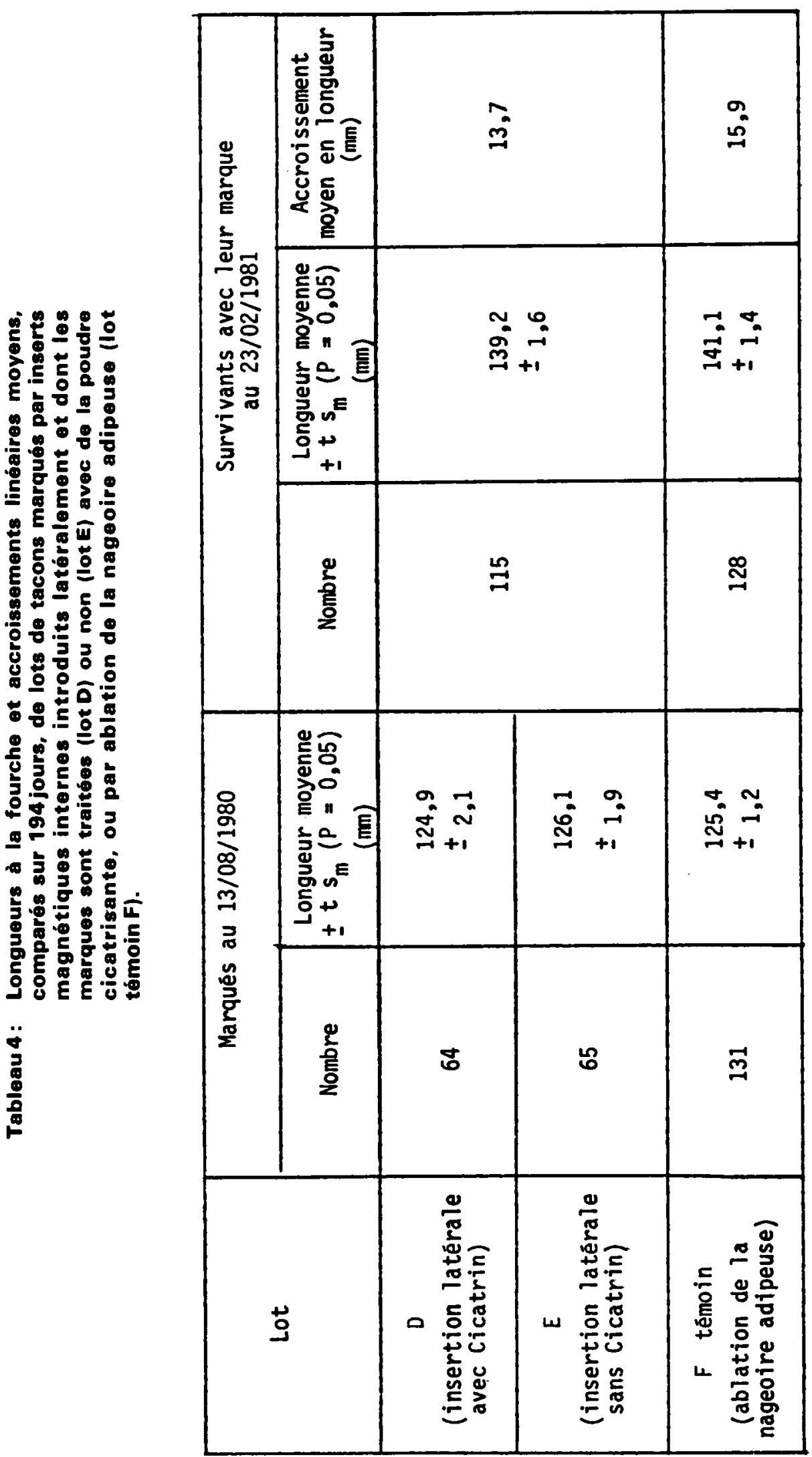
BERGMAN, 1977). Ainsi, l'immersion du dispositif de décodage, constitué d'une boucle de solénoïde, dans un passage obligé, tel une échelle à poissons pour les contrôles en milieu naturel, permet l'identification et l'enregistrement du moment du passage des sujets marqués sans aucune manipulation; ceci constitue un progrès technique considérable.

Ce marquage est particulièrement recommandé pour les observations successives des mêmes lots de poissons à différentes phases de leur cycle (ex.: marquage de tacons originaires de plusieurs secteurs de rivière, vérifiés au stade saumoneau, lors de leur avalaison et à nouveau contrôlés à leur retour dans des passes à poissons pourvues de détecteurs-décodeurs immergeables).

Le prix de l'appareil de détection (version non immergeable) est de $41000 \mathrm{~F}$ en 1982 , celui des marques est de $3 \mathrm{~F}$ pièce. Ce système de marquage expérimental est peu coûteux en regard des avantages qu'il procure.

\section{BIBLIOGRAPHIE}

ANONYME, 1979. Procédé de marquage magnétique interne pour poissons. Journées Aquaculture extensive et repeuplement, C.N.E.X.O., Brest, 29-31 mai, 3 p.

CARLIN B., 1955. Tagging of salmon smolts in the river Lagan. Rep. short Pap. Inst. Freshwat. Res. Drottningholm. 1954, 36, 57-74.

DUMAS J., 1977. Cryomarquage : caractéristiques d'un appareil et essai sur de jeunes saumons atlantiques (Salmo salar L.). Bull. Fr. Piscic., 267, 41-61.

DUMAS J., 1978. Premières observations de cryomarques sur les adultes de saumons atlantiques (Salmo salar L.) de la Nivelle marqués au stade smolt. Bull. Fr. Piscic., 270. 218-222.

HARACHE Y., LAGARDE P., PROUZET P., 1978. Essais d'une marque magnétique interne pour smolts de saumons. Saumons, 26, 31-36.

ISAKSSON A., BERGMAN P.K., 1977. Evaluation of two tagging methods used to study the efficiency of Atlantic salmon hatchery operations in Iceland. I.C.E.S., Anad. Catad. Fish. Comm. C.M. 1977/M: 36, 11 p.

PIGGINS J.D., 1972. Cold branding as smolt marking technique. J. Inst. Fish. Mgmt, 3, 9-11.

REFSTIE T., AULSTAD D., 1975. Tagging experiments with salmonids. Aquaculture, 5 (4), 367-374.

SAUNDERS R.L., ALLEN K.R., 1967. Effect of tagging and fin-clipping on the survival and growth of Atlantic salmon between smolt and adult stages. J. Fish. Res. Bd Can., 24 (12), 2595-2611. 